\title{
Complex neuromuscular disorder in a patient with chronic myeloid leukaemia
}

\author{
Peter S. Bergin and Andrew N. Gale
}

Royal Free Hospital, Pond Street, London NW3 2QG, UK

\begin{abstract}
Summary: We report a 44 year old man who developed external ophthalmoplegia and predominantly respiratory, truncal and bulbar weakness with brisk reflexes, histological evidence of an inflammatory myopathy and a high titre of acetylcholine receptor antibodies, one month after starting hydroxyurea and allopurinol for chronic myeloid leukaemia. The temporal relationship suggests a possible association between this patient's unusual neuromuscular disorder and either the chronic myeloid leukaemia or its treatment.
\end{abstract}

\section{Introduction}

A variety of neurological disorders occur in patients with acute leukaemia, ${ }^{1}$ though neurological complications of chronic myeloid leukaemia are uncommon. We report here a patient with a previously undescribed combination of ophthalmoplegia, myositis and pyramidal and bulbar signs developing one month after a diagnosis of chronic myeloid leukaemia (CML).

\section{Case report}

In early May 1989, a previously healthy 44 year old man was found to have a blood white cell count of $221 \times 10^{9} / 1$ when he presented for routine blood donation. A diagnosis of chronic myeloid leukaemia was confirmed by bone marrow aspirate, and cytogenetic studies revealed a Philadelphia chromosome; hydroxyurea $1 \mathrm{~g}$ twice daily and allopurinol $300 \mathrm{mg}$ daily were commenced. He was admitted urgently on 8 June 1989 with rapidly increasing weakness and shortness of breath. He had a long-standing divergent squint. Neurological examination revealed total external ophthalmoplegia with bilateral ptosis and normal pupils. There was severe weakness of trunk flexion and proximal lower limb weakness, but no wasting or fasciculations and no fatiguability. Plantar

Correspondence: P.S. Bergin, M.B., Ch.B., Department of Clinical Neurophysiology, National Hospital for Neurology and Neurosurgery, Queen Square, London WC1N 3BG, UK.

Accepted: 22 September 1992 responses were flexor, and upper limb tendon jerks were normal, but knee and ankle jerks were very brisk $(3+)$. Clonus was not present. No jaw jerk could be elicited, and abdominal reflexes were absent. There was no sensory loss or bulbar involvement at that time, but he had shallow respirations with paradoxical abdominal movements. Blood gas analysis confirmed that he was in respiratory failure and he required artifical ventilation. At no time had he ever received penicillamine.

Chest X-ray revealed a high diaphragm but no pulmonary lesion. The blood white cell count was $158 \times 10^{9} / 1$, the haemoglobin $11.3 \mathrm{~g} / 1$ and platelet count $508 \times 10^{9} / 1$. Blood biochemistry was normal apart from an elevated creatine kinase (CK) at 1,200 units/1 (normal <200). Computed tomographic (CT) scans of the head and mediastinum were normal, and CT of the orbits revealed slender extraocular muscles, but no other abnormality. Cerebrospinal fluid (CSF) analysis on admission and 3 weeks later was normal, including negative cytology and negative test for oligoclonal bands. Botulinum toxin was not detected in serum, gastric aspirate or stool. Acetylcholine receptor antibodies were present at a titre of $7 \times 10^{-10} \mathrm{mcl} / 1$ on admission and 2 weeks later the titre had risen to $106 \times 10^{-10} \mathrm{~mol} / \mathrm{l}$. Anti-striated muscle antibodies were present at a titre of $1 / 40$, but autoantibodies against thyroid and nuclear antigens including extractable nuclear antigens and RNA synthetases were not detected. No assay for anti-GM $\mathbf{M}_{1}$ antibodies was performed.

Shortly after admission edrophonium $20 \mathrm{mg}$ was administered with no benefit. Four further edrophonium tests were performed between June 
and November 1989 and on no occasion was there any objective improvement.

Nerve conduction studies and electromyography (EMG) were performed on 8 June, 27 June and 28 July 1989. Studies included repetitive nerve stimulation and single fibre EMG. Initial studies were normal, but subsequent EMG revealed minor myopathic changes (scattered fibrillations and positive sharp waves, and some short duration polyphasic units). At no time was excessive jitter or blocking noted, and repetitive stimulation was normal. Nerve conduction (including $F$ waves) remained normal.

Biopsy of left tibialis anterior (5 July 1989) revealed a florid inflammatory infiltrate, necrotic fibres and multiple internally placed sarcolemmal nuclei. The infiltrate comprised mainly macrophages, but immunological studies demonstrated the presence of $T$ and $B$ lymphocytes. There was no evidence of vasculitis.

He developed increasing limb and trunk weakness with particular weakness of neck and bulbar muscles, atrophy of the tongue, and aspiration of fluid on swallowing. No fasciculations of the tongue were seen.

He was treated with prednisolone $100 \mathrm{mg}$ per day for the first month, with lower doses for 6 months. He had two courses of high dosage intravenous methylprednisolone, a course of plasmapheresis, and immunosuppression with azathioprine followed by cyclophosphamide. Pyridostigmine was commenced after the result of the first acetylcholine receptor antibody assay became available. Thymectomy was considered but it was felt that the risks of the procedure outweighed the likely benefits. Hydroxyurea was discontinued 2 weeks after admission when the blood white cell count fell to $12.1 \times 10^{9} / 1$. In mid-July 1989 the peripheral white count rose to $35 \times 10^{9} / 1$ and he responded to two doses of busulphan $50 \mathrm{mg} 10$ days apart. No further doses of busulphan or hydroxyurea were required and his blood white cell count remained normal, but the Philadelphia chromosome continued to be present in myeloid cells.

He required artificial ventilation until June 1990 when he became able to breathe spontaneously for periods of up to 2 hours/day. There was slight improvement in extrocular movements and limb power but trunk muscles remained very weak and he still required nasogastric feeding. Lower limb reflexes remained exaggerated. The acetylcholine receptor antibody titre in June 1990 had fallen to the borderline normal range at $4 \times 10^{-10} \mathrm{mmol} / 1$ and anti-striated muscle antibodies had become negative.

Between June 1990 and January 1992 both his neurological condition and his CML remained stable. He continued to have profound weakness of trunk muscles and markedly restricted eye movements. His respiratory function slowly improved so that by the end of 1991 he was able to breathe spontaneously for up to 8 hours, though it was not possible to wean him completely from the ventilator. During this period he received no specific treatment for either his CML or neurological illness, apart from pyridostigmine. In January 1992 he developed an acute myeloblastic transformation which was rapidly fatal. Unfortunately, the relatives would not agree to a postmortem being performed.

\section{Discussion}

This patient's neuromuscular disturbance does not fit easily into any single disease entity. He had an illness affecting purely motor systems with features suggesting an associated brainstem lesion. Combined lower and upper motor neurone signs are features of amyotrophic lateral sclerosis, but the eventual improvement argues strongly against this diagnosis. There was histological evidence of a myositis, and the CK was elevated on admission. However, external ophthalmoplegia does not occur in polymyositis. It is common in myasthenia gravis, and occasional patients with clinical and pathological features of both polymyositis and myasthenia gravis have been reported. ${ }^{2,3}$ Acetylcholine receptor antibodies in a titre of $106 \times 10^{-10} / 1$ would generally be considered diagnostic of myasthenia gravis ${ }^{4}$ but we were unable to demonstrate abnormalities on repetitive nerve stimulation or single fibre EMG, and there was no improvement during treatment with anti-cholinesterase drugs, plasmapheresis or immunosuppression. Acute ophthalmoplegia and respiratory failure occur in the Miller Fisher syndrome but myositis is not a feature of this condition, reflexes are usually lost rather than exaggerated, and CSF protein is usually elevated.

We are therefore unable to give a clear label to this patient's complex neuromuscular disorder. In addition, it is not clear how the neuromuscular disorder relates to his CML. The CML also followed an unusual course because it remained in remission without treatment for over 2 years. While it is possible that the occurrence of the two illnesses within a month of each other is a coincidence, we think this is unlikely. He had previously been in excellent health, and was entirely asymptomatic when he started treatment for the CML. The presence of acetylcholine receptor and anti-striated muscle antibodies, together with the myositis suggest an autoimmune process, despite the lack of any obvious response to treatment. Since CML is a malignancy of the granulocyte line, immunological disturbances are not usually a feature. However, 
there are reports of dermatomyositis complicating $\mathrm{CML}^{5,6}$ and of CML and myasthenia gravis occurring together. ${ }^{7-9}$ There are some similarities between our patient and a patient reported by Djaldetti $e t$ $a l^{8}$ A 70 year old man developed diplopia, limb and pharyngeal weakness, and respiratory difficulties 5 years after a diagnosis of CML had been made, and for which he had been treated with busulphan. A diagnosis of myasthenia gravis was made after a positive Tensilon test, and gradual improvement followed a thymectomy. However, for the reasons given above we do not think a diagnosis of myasthenia gravis can satisfactorily explain our patient's neurological problems. Our patient differs from Djaldetti's in having increased reflexes, an atrophied tongue, an elevated CK and biopsyproven myositis.

The possibility that the neuromuscular disorder may have been secondary to treatment for CML also needs to be considered. Djaldetti et al. speculated that the myasthenia gravis in their patient may have been induced by busulphan. Busulphan clearly cannot be responsible for our patient's neurological problems as he never received the drug until well after the neurological illness developed. He had received hydroxyurea and allopurinol. Allopurinol seems an unlikely cause. It has been used extensively for many years

\section{References}

1. Wolk, R., Masse, S., Conklin, R. \& Friereich, E. The incidence of central nervous system leukemia in adults with acute leukemia. Cancer 1979, 33: 863-869.

2. Namba, T., Brunner, D. \& Grob, D. Association of myasthenia gravis with pemphigus vulgaris, candida albicans infection, polymyositis and myocarditis. J Neurol Sci 1973, 20: 231-242.

3. Vasilecu, C., Bucur, G., Petrovici, A. \& Florescu, A. Myasthenia in patients with dermatomyositis. J Neurol Sci 1979, 38: $129-144$.

4. Lindstrom, J. An assay for antibodies to human acetylcholine receptor in serum from patients with myasthenia gravis. Clin Immunol Immunopathol 1977, 7: 36-43.

5. Gascard, E., Regis, M., Moulard, J. \& Muller, G. Dermatomyosite et leucemie myeloide. Marseille Med 1969, 106: 329-331.

6. Tierney, L., Jensen, B. \& Schwartz, R. Dermatomyositis associated with chronic myelogenous leukaemia. Dermatologica 1985, 171: 189-192.

7. Sparagana, M., Grieble, H. \& Rubenstein, H. Granulocytic leukemia associated with myxedema, thyroiditis and myasthenia gravis. J Am Geriatr Soc 1972, 20: 272-278.

8. Djaldetti, M., Pinkhas, J., de Vries, A., Kott, E., Joshua, H. \& Dolberg, L. Myasthenia gravis in a patient with chronic myeloid leukemia treated by busulfan. Blood 1968, 32: 336-340. during which time no case resembling ours has ever been reported, and there seems little theoretical reason to invoke it. Hydroxyurea interferes with DNA synthesis by inactivating the enzyme ribonucleotide diphosphate reductase. This enzyme catalyses the reductive conversion of ribonucleotides to doxyribonucleotides, and is thought to be a rate-limiting step in DNA synthesis. ${ }^{10}$ Toxicity from hydroxyurea usually involves reversible myelosuppression, and we are not aware of neuromuscular problems being previously reported following its use. Although autoimmune illnesses are not commonly associated with hydroxyurea, it clearly does have major effects on the immune system. In mice, intraperitoneal injection induces a marked depletion of both $T$ and $B$ cells in peripheral and central lymphoid organs $\mathrm{s}^{11,12}$ and an altered pattern of antibody responses. ${ }^{13}$ The latter is at least in part the result of a selective action on $T$ helper cells. ${ }^{13}$ Human $T$ lymphocytes stimulated with phytohaemagglutinin (PHA) in vitro increase in numbers of interleukin 2 receptors following treatment with hydroxyurea ${ }^{14}$ We suggest, therefore, that treatment with hydroxyurea may have altered the balance between this patient's $T$ suppressor and $T$ helper lymphocytes with subsequent development of an autoimmune condition producing this complex neurological disorder.

9. Wanders, J., Wattendorff, A., Endte, L., den Nijs, J. \& Leeksma, C. Chronic myeloid leukaemia in myasthenia gravis after long term treatment with 6-mercaptopurine. Acta Med Scand 1981, 210: 235-238.

10. Donehower, R. Hydroxyurea. In: Chabner, B. \& Collins, J. (eds) Cancer Chemotherapy: Principles and Practice. Lippincott, Philadelphia, 1990.

11. Freitas, A., Rocha, B., Forni, L. \& Coutinho, A. Population dynamics of B lympocytes and their precursors: demonstration of high turnover in the central and peripheral lymphoid organs. J Immunol 1982, 128: 54-60.

12. Rocha, B., Freitas, A. \& Coutinho, A. Population dynamics of $T$ lymphocytes. Renewal rate and expansion in the peripheral lymphoid organs. J Immunol 1983, 131: 2158-2164.

13. Cumano, A., Vieira, P., Colle, J., Truffa-Bachi, P. \& Freitas, A. Effects of hydroyxurea in vivo treatment on the antibody response in mice. Ann Inst Pasteur/Immunol 1986, 137: 355-367.

14. Depper, J., Leonard, W., Drogula, C., Kronke, M., Waldmann, T. \& Greene, W. Activators of protein kinase $C$ and 5-Azacytidine induce IL-2 receptor expression on human T lymphocytes. J Cell Biochem 1985, 27: 267-276. 